\title{
Meningite tuberculosa: avaliação do desempenho do Xpert MTB/RIF Ultra para o
}

\section{diagnóstico}

\author{
Tuberculous meningitis: performance assessment of Xpert MTB/RIF Ultra for diagnosis \\ Meningitis tuberculosa: evaluación del rendimiento de Xpert MTB/RIF Ultra para el diagnóstico
}

Recebido: 11/02/2022 | Revisado: 19/02/2022 | Aceito: 23/02/2022 | Publicado: 05/02/2022

Savyla Franciele Soares Silva

ORCID: https://orcid.org/0000-0002-5584-381X Laboratório Estadual de Saúde Pública Dr. Giovanni Cysneiros, Brasil Hospital Estadual de Doenças Tropicais Dr. Anuar Auad, Brasil E-mail: savyla_ss1@hotmail.com

Camila Aparecida Nunes de Albuquerque ORCID: https://orcid.org/0000-0001-5241-8781 Laboratório Estadual de Saúde Pública Dr. Giovanni Cysneiros, Brasil Hospital Estadual de Doenças Tropicais Dr. Anuar Auad, Brasil E-mail: camillaalbuquerque99@gmail.com

Sueli Lemes de Ávila Alves

ORCID: https://orcid.org/0000-0001-8608-6658 Laboratório Estadual de Saúde Pública Dr. Giovanni Cysneiros, Brasil E-mail: suelilemesgyn@gmail.com

Heberson Alves de Oliveira

ORCID: https://orcid.org/0000-0002-8538-2153 Laboratório Estadual de Saúde Pública Dr. Giovanni Cysneiros, Brasil E-mail: hebersonbusiness@gmail.com

Rhalcia Cristina de Melo Lima ORCID: https://orcid.org/0000-0003-2921-1867 Laboratório Estadual de Saúde Pública Dr. Giovanni Cysneiros, Brasil E-mail: rhalcialima17@gmail.com

Edna Joana Cláudio Manrique ORCID: https://orcid.org/0000-0002-8632-3542 Laboratório Estadual de Saúde Pública Dr. Giovanni Cysneiros, Brasil Residência Multiprofissional em Infectologia HDT/LACEN-GO. Brasil Escola de Ciências Médicas e da Vida, Brasil E-mail: ednamanrique@gmail.com

\begin{abstract}
Resumo
Objetivo: Avaliar o desempenho diagnóstico do Xpert MTB/RIF Ultra para meningite tuberculosa em amostras de líquido cefalorraquidiano. Trata-se de um estudo do tipo transversal quantitativo retrospectivo desenvolvido a partir de informações obtidas dos registros da seção de Micobactérias do Laboratório Estadual de Saúde Pública Dr. Giovanni Cysneiros - LACEN-GO no período de novembro de 2019 a janeiro de 2021. Foram calculados os valores de sensibilidade, especificidade, valor preditivo positivo (VPP) e valor preditivo negativo (VPN) e seus respectivos intervalos de confiança de $95 \%$. Um total de 247 amostras de líquido cefalorraquidiano foram incluídas no estudo no período analisado, sendo que houve crescimento do Complexo Mycobacterium tuberculosis (CMT) na cultura sólida em duas $(0,8 \%)$ amostras e o Xpert MTB/RIF Ultra detectou 11 (4,5\%) amostras com material genético e de acordo com a técnica os resultados obtidos foram classificados em: traços $(n=6)$, muito baixo $(n=1)$ e baixo $(n=4)$. Ao avaliar o desempenho do Xpert verificou a sensibilidade de 100\% (IC 95\% 16-100), especificidade de 96\% (IC 95\% 93-98), VPP 18\% (IC 95\% 18-30) e VPN de 100\% (IC 95\% 100-100). Em conclusão, o Xpert MTB/RIF Ultra apresentou uma sensibilidade e especificidade alta para o diagnóstico de meningite tuberculosa, apesar desta última ter sido ligeiramente reduzida. Esses resultados demonstram que o Xpert MTB/RIF Ultra apresenta melhorias que contribuem significativamente para a identificação dos casos de meningite tuberculosa.
\end{abstract}

Palavras-chave: Meningite tuberculosa; Líquido cefalorraquidiano; Diagnóstico.

\footnotetext{
Abstract

Objective: To evaluate the diagnostic performance of Xpert MTB/RIF Ultra for tuberculous meningitis in cerebrospinal fluids samples. Methodology: This is a retrospective quantitative cross-sectional study developed from information obtained from the records of the Mycobacteria section of the Laboratório Estadual de Saúde Pública Dr. Giovanni Cysneiros - LACEN-GO from November 2019 to January 2021. Sensitivity, specificity, positive predictive value (PPV) and negative predictive value (NPV) and their respective 95\% confidence intervals were calculated. Results: A total of 247 cerebrospinal fluid samples were included in the study during the period analyzed, with
} 
Mycobacterium tuberculosis Complex (MTC) growing in solid culture in two (0.8\%) samples and Xpert MTB/RIF Ultra detected $11(4.5 \%)$ samples with genetic material and, according to the technique, the results obtained were classified into: trace $(n=6)$, very low $(n=1)$ and low $(n=4)$. When evaluating the performance of Xpert, the sensitivity was $100 \%$ (95\% CI 16-100), 96\% specificity (95\% CI 93-98), 18\% PPV (95\% CI 18-30) and 100\% NPV (CI 95\% 100-100). Conclusion: Xpert MTB/RIF Ultra had a high sensitivity and specificity for the diagnosis of tuberculous meningitis, although the latter was been slightly reduced. These results demonstrate that Xpert MTB/RIF Ultra presents improvements that significantly contribute to the identification of cases of tuberculous meningitis.

Keywords: Tuberculous meningitis; Cerebrospinal fluid; Diagnosis.

\section{Resumen}

Objetivo: Evaluar el rendimiento diagnóstico de Xpert MTB / RIF Ultra para la meningitis tuberculosa en muestras de líquido cefalorraquídeo. Se trata de un estudio transversal cuantitativo retrospectivo elaborado a partir de información obtenida de los registros de la sección de Micobacterias del Laboratório Estadual de Saúde Pública Dr. Giovanni Cysneiros - LACEN-GO de noviembre de 2019 a enero de 2021. Sensibilidad, especificidad, valor predictivo positivo (VPP) y valor predictivo negativo (VPN) y sus respectivos intervalos de confianza del 95\% se calcularon. Se incluyeron en el estudio un total de 247 muestras de líquido cefalorraquídeo durante el período analizado, con Complejo Mycobacterium tuberculosis (CMT) creciendo en cultivo sólido en dos (0,8\%) muestras y Xpert MTB / RIF Ultra detectó $11(4,5 \%)$ muestras con material genético y, según la técnica, los resultados obtenidos se clasificaron en: rasgos $(\mathrm{n}=6)$, muy bajos $(\mathrm{n}=1)$ y bajos $(\mathrm{n}=4)$. Al evaluar el rendimiento de Xpert, la sensibilidad fue del $100 \%$ (95\% CI 16-100), 96\% de especificidad (95\% CI 93-98), 18\% PPV (95\% CI 18-30) y 100\% VPN (CI 95\% 100-100). En conclusión, Xpert MTB / RIF Ultra tuvo una alta sensibilidad y especificidad para el diagnóstico de meningitis tuberculosa, aunque este último se ha reducido ligeramente. Estos resultados demuestran que Xpert MTB / RIF Ultra presenta mejoras que contribuyen significativamente a la identificación de casos de meningitis tuberculosa.

Palabras clave: Meningitis tuberculosa; Líquido cefalorraquídeo; Diagnóstico.

\section{Introdução}

A tuberculose é considerada um problema de saúde pública mundial por ser uma doença infectocontagiosa generalizada grave que apresenta elevada morbidade e mortalidade. A tuberculose é causada por micobactérias pertencentes ao Complexo Mycobacterium tuberculosis (CMT), constituído por diversas espécies como: M. tuberculosis, Mycobacterium bovis, Mycobacterium africanum e Mycobacterium microti, sendo o M. tuberculosis a espécie de maior relevância na saúde pública (Zhang et al., 2017; World Health Organization, 2021).

A doença acomete geralmente os pulmões, porém pode afetar outros órgãos e sistemas do corpo, como os rins, ossos e o Sistema Nervoso Central (SNC), sendo chamada de tuberculose extrapulmonar. A meningite tuberculosa é um tipo de tuberculose extrapulmonar grave que se desenvolve no SNC e que pode apresentar desfechos desfavoráveis e levar à morte ou gerar incapacidades em parte das pessoas afetadas. É caracterizada por uma inflamação subaguda ou crônica das meninges devido à disseminação do M. tuberculosis no espaço subaracnoide. Essa forma de tuberculose é na maioria dos casos derivada de uma complicação da tuberculose pulmonar e surge normalmente nos primeiros seis meses após o indivíduo ser infectado. Não apresenta transmissibilidade, a menos que o indivíduo apresente tuberculose pulmonar concomitante, permanecerá transmissível enquanto a doença pulmonar estiver ativa (Seddon et al., 2019; Cresswell et al., 2020; Garcia et al., 2016).

A meningite tuberculosa acomete principalmente crianças e indivíduos portadores de HIV e quando não tratada é completamente fatal. Mesmo diante de um diagnóstico e tratamento rápido, em torno de um quinto dos indivíduos morrem e a maioria dos que sobrevivem apresentam problemas neurológicos significativos. De acordo com a Organização Mundial da Saúde (OMS) é estimado a cada ano cerca de 10,4 milhões de casos novos de tuberculose e é sugerido que no mínimo 100.000 indivíduos desenvolvam meningite tuberculosa anualmente, porém esse número pode ser bem maior visto que muitos casos não são diagnosticados, devido à deficiência no desempenho dos testes diagnósticos (Seddon et.al, 2019; Seddon et al., 2019).

Esta doença apresenta difícil confirmação por critérios microbiológicos, principalmente por ser uma patologia paucibacilar, ou seja, que apresenta baixa carga bacilar, o que dificulta o isolamento do microrganismo no LCR por metodologias convencionais. Diante disso, algumas mudanças foram necessárias para aprimorar o diagnóstico da meningite 
tuberculosa e métodos genotípicos como o Teste Rápido Molecular para a tuberculose (TRM-TB) estão sendo utilizados para possibilitar a identificação de forma mais rápida da meningite tuberculosa (Garg, 2019).

Em 2013, a OMS estabeleceu TRM-TB como a melhor metodologia de triagem para meningite tuberculosa. O TRMTB é uma tecnologia que permite a amplificação de ácidos nucleicos utilizados na identificação do M. tuberculosis e possibilita a detecção de mutações em genes que estão associados à resistência à rifampicina. Esse teste genotípico apresenta maior rapidez na liberação do resultado, além de sensibilidade e especificidade mais elevadas (Cresswell et al., 2020).

A metodologia GeneXpert ${ }^{\circledR}$ MTB/RIF da Cepheid é um TRM-TB indicado pela OMS que permite o diagnóstico de tuberculose em aproximadamente duas horas. Porém, estudos realizados apontaram que o Xpert MTB/RIF apresenta um desempenho adequado para o diagnóstico de tuberculose pulmonar, entretanto tem uma baixa sensibilidade em algumas amostras extrapulmonares paucibacilares (WU et al., 2019). A fim de resolver essa limitação relacionada ao Xpert MTB/RIF foi desenvolvido um novo ensaio na mesma plataforma GeneXpert, denominado Xpert MTB/RIF Ultra buscando aumentar a sensibilidade para a detecção do M. tuberculosis e da resistência à rifampicina. (Chin et al., 2019a; Chakravorty et al., 2017).

Por ser um teste que começou a ser utilizado recentemente, ainda há poucos estudos que avaliam esse novo ensaio, principalmente para meningite tuberculosa. Dessa forma, o presente estudo torna-se fundamental para acrescentar mais conhecimento acerca do Xpert MTB/RIF Ultra. Diante disso, o objetivo do estudo foi avaliar o desempenho diagnóstico do Xpert MTB/RIF Ultra para meningite tuberculosa em amostras de líquor.

\section{Metodologia}

Trata-se de um estudo observacional quantitativo do tipo transversal analítico e retrospectivo desenvolvido a partir de informações obtidas dos registros da seção de Micobactérias do Laboratório Estadual de Saúde Pública Dr. Giovanni Cysneiros - LACEN-GO, responsável por realizar o diagnóstico para hanseníase, micobactérias tuberculosas e não tuberculosas. O presente estudo utilizou como subsídio de metodologia de pesquisa científica as orientações de Pereira, Shitsuka, Parreira \& Shitsuka, (2018).

Foram incluídos na pesquisa todos os resultados do Xpert MTB/RIF Ultra e cultura sólida do líquor em meio Lowenstein Jensen para meningite tuberculosa, realizados na mesma amostra, contidos nos registros da seção de Micobactérias do LACEN-GO, no período de novembro de 2019 a janeiro de 2021. Foram excluídos do estudo todos os resultados das culturas para meningite tuberculosa que apresentaram somente contaminantes como bactérias que não fazem parte do Complexo M. Tuberculosis e fungos e aquelas cujas amostras foram insuficientes para a realização das duas metodologias.

Os dados foram tabulados no programa Microsoft Office Excel 2013®, sendo expressos como valores de frequência absoluta e relativa. Por meio do software MedCalc ${ }^{\circledR}$ versão 19.5.3 foram calculados os valores de sensibilidade, especificidade, valor preditivo positivo e valor preditivo negativo e seus respectivos intervalos de confiança de $95 \%$ a fim de avaliar o desempenho da metodologia.

A cultura sólida em meio Lowenstein Jensen foi categorizada em negativa e positiva, conforme a quantidade de colônias no meio de cultura. Já para o Xpert MTB/RIF Ultra os resultados foram classificados em não detectado e detectado: traços, muito baixo, baixo, médio e alto.

Conforme regulamenta a Resolução n ${ }^{\circ}$ 466/2012 do Conselho Nacional de Saúde (CNS) e normas complementares, as pesquisas que envolvem seres humanos devem ser submetidas ao Comitê de Ética em Pesquisa (CEP), desta forma este estudo foi submetido ao CEP, sendo aprovado com número de parecer 4.605.062. 


\section{Resultados}

Um total de 247 amostras de líquor foram incluídas no estudo no período analisado, sendo que houve crescimento do Complexo Mycobacterium tuberculosis na cultura sólida em duas (0,8\%) amostras e o Xpert MTB/RIF Ultra detectou 11 $(4,5 \%)$ amostras com material genético. Portanto, o TRM detectou nove amostras positivas que se apresentaram negativas na cultura. Foram negativas na cultura e no Xpert MTB/RIF Ultra respectivamente, 245 (99,2\%) e 236 (95,5\%) amostras (Tabela $1)$.

Tabela 1. Frequências absolutas e relativas dos resultados do Xpert MTB/RIF Ultra e da cultura sólida $(\mathrm{n}=247)$.

\begin{tabular}{|l|l|l|}
\hline \multicolumn{1}{|c|}{ Resultados } & \multicolumn{1}{|c|}{ Cultura Sólida n (\%) } & \multicolumn{1}{c|}{ Xpert MTB/RIF Ultra n (\%) } \\
\hline Positivo/ Detectado & $2(0,8)$ & $11(4,5)$ \\
\hline Negativo/Não detectado & $245(99,2)$ & $236(95,5)$ \\
\hline Total & $247(100)$ & $247(100)$ \\
\hline
\end{tabular}

Fonte: Autores.

O Xpert MTB/RIF Ultra identificou 11 amostras positivas para o Complexo Mycobacterium tuberculosis e de acordo com a metodologia utilizada os resultados obtidos foram classificados em: traços $(n=6)$, muito baixo $(n=1)$ e baixo $(n=4)$. Nenhuma amostra se enquadrou nas categorias médio e alto (Tabela 2).

Ao correlacionar os resultados do Xpert MTB/RIF Ultra com a cultura sólida foi possível observar que nove $(81,8 \%)$ amostras apresentaram resultado detectado no Xpert MTB/RIF Ultra sendo negativas na cultura, destas, seis (54,5\%) foram classificadas como traços, uma $(9,1 \%)$ muito baixo e duas $(18,2 \%)$ baixo (Tabela 2$)$.

Duas amostras apresentaram resultados positivos nos dois testes, ambas foram classificadas como baixo no Xpert MTB/RIF Ultra, das quais uma $(9,1 \%)$ apresentou crescimento de duas colônias na cultura e uma $(9,1 \%)$ crescimento de 15 colônias (Tabela 2).

Tabela 2. Correlação entre os resultados positivos $(\mathrm{n}=11)$ do Xpert MTB/RIF Ultra e cultura sólida.

\begin{tabular}{|c|c|c|c|c|}
\hline \multicolumn{2}{|c|}{ Cultura Sólida } & \multicolumn{4}{|c|}{ Xpert MTB/RIF Ultra Detectado } \\
\hline & $\begin{array}{c}\text { Traços } \\
\mathbf{n}(\%)\end{array}$ & $\begin{array}{c}\text { Muito baixo } \\
\mathbf{n}(\%)\end{array}$ & $\begin{array}{c}\text { Baixo } \\
\text { n (\%) }\end{array}$ & $\begin{array}{c}\text { Total } \\
\mathbf{n}(\%)\end{array}$ \\
\hline & $6(54,5)$ & $1(9,1)$ & $2(18,2)$ & $9(81,8)$ \\
\hline Negativo & 0 & 0 & $1(9,1)$ & $1(9,1)$ \\
\hline Positivo 2 Colônias & 0 & 0 & $1(9,1)$ & $1(9,1)$ \\
\hline Positivo 15 Colônias & $6(54,5)$ & $1(9,1)$ & $4(36,4)$ & $11(100 \%)$ \\
\hline
\end{tabular}

Fonte: Autores.

Ao avaliar o desempenho do Xpert MTB/RIF Ultra considerando a cultura sólida como metodologia padrão-ouro, verificou-se a sensibilidade de $100 \%$ (IC 95\% 16-100) e especificidade de $96 \%$ (IC 95\% 93-98). O valor preditivo positivo (VPP) encontrado foi de 18\% (IC 95\% 18-30) e o valor preditivo negativo (VPN) de 100\% (IC 95\% 100-100) (Tabela 3 ). 
Tabela 3. Desempenho diagnóstico do Xpert MTB/RIF Ultra comparado à cultura sólida para a detecção de meningite tuberculosa.

\begin{tabular}{|l|l|}
\hline Variáveis & $\begin{array}{l}\text { Detecção do Complexo Mycobacterium tuberculosis } \\
\%(\text { IC 95\%) }\end{array}$ \\
\hline Sensibilidade & $100(16-100)$ \\
\hline Especificidade & $96(93-98)$ \\
\hline VPP & $18(10-30)$ \\
\hline VPN & $100(100-100)$ \\
\hline
\end{tabular}

VPP: Valor Preditivo Positivo; VPN: Valor Preditivo Negativo. Fonte: Autores.

\section{Discussão}

A meningite tuberculosa é uma emergência médica e o diagnóstico rápido e início do tratamento específico podem ser decisivos no prognóstico, porém, devido à natureza paucibacilar da doença, o seu diagnóstico se torna muitas vezes desafiador. Com os avanços recentes nos testes moleculares, esse diagnóstico está apresentando melhorias consideráveis para aumentar o desempenho do teste (Huang et al., 2021; Shao et al., 2020).

O Xpert MTB/RIF Ultra pode ser considerada uma metodologia útil para o diagnóstico definitivo da meningite tuberculosa antes que ocorram as sequelas irreversíveis. Esse teste molecular foi desenvolvido como um ensaio de nova geração a fim de superar algumas limitações encontradas na sua versão anterior, principalmente para amostras paucibacilares. Avanços significativos podem ser observados no Xpert MTB/RIF Ultra, como o aumento da capacidade total do cartucho e inclusão de dois alvos de amplificação diferentes, resultando em diminuição do limiar de detecção das unidades formadoras de colônias bacterianas - UFC (Huang et al., 2021; Bisognin et al., 2018; Chin et al., 2019b).

O presente estudo avaliou o desempenho do ensaio molecular Xpert MTB/RIF Ultra no diagnóstico de meningite tuberculosa em amostras de líquor. Os dados demonstraram que o Xpert MTB/RIF Ultra apresentou sensibilidade de 100\%, especificidade $96 \%$, VPP $18 \%$ e VPN $100 \%$.

A sensibilidade desse estudo foi maior quando confrontado com os estudos publicados (Shao et al., 2020; Bahr et al., 2018). Isso pode ser explicado pelo fato do estudo em questão utilizar como padrão ouro a cultura para micobactérias, diferentemente dos estudos encontrados na literatura que apresentaram variação quanto ao padrão de referência, sendo utilizada a microscopia e/ou achados de imagem e/ou qualquer positividade de teste no líquor por cultura e/ou PCR. Todavia a cultura foi escolhida como método para comparação no presente estudo por ser consagrada como padrão ouro para tuberculose (Costa et al., 2018).

Apesar disso, observou-se a sensibilidade aumentada no estudo em questão e nos estudos analisados. Em uma pesquisa multicêntrica prospectiva conduzida na China, os dados demonstraram que o Xpert MTB/RIF Ultra apresentou uma sensibilidade de 93,3\%, ao utilizar como referência a evidência microbiológica por cultura e/ou Xpert MTB/RIF Ultra. De forma semelhante, um estudo prospectivo realizado em Uganda em pacientes infectados pelo HIV revelou que o Xpert MTB/RIF Ultra apresentou 95\% de sensibilidade para o diagnóstico de casos de meningite tuberculosa definitiva (Shao et al., 2020; Bahr et al., 2018).

Conforme dados reportados na literatura, um teste diagnóstico com sensibilidade acima de $90 \%$ para meningite tuberculosa e que permite ter o resultado em menos de 90 minutos, apresenta elevado potencial para melhorar o diagnóstico dos pacientes, principalmente para detectar paucibacilares. Essa sensibilidade aumentada no Xpert MTB/RIF Ultra pode ser atribuída principalmente ao seu limite de detecção extrassensível em torno de $10 \mathrm{UFC} / \mathrm{mL}$ em comparação com cerca de 100 UFC/mL do Xpert MTB/RIF. Apesar do limite de detecção do Xpert MTB/RIF Ultra ser semelhante ao da cultura líquida (10 $\mathrm{UFC} / \mathrm{mL}$ ), a sensibilidade desse novo ensaio mostra-se mais aprimorada, devido provavelmente à capacidade de detectar bacilos não viáveis, fato que não ocorre na cultura (Bahr et al., 2018; Chakravorty et al., 2017). 
No estudo em questão, o valor da especificidade foi ligeiramente menor do que os valores identificados em trabalhos publicados anteriormente. A pesquisa multicêntrica prospectiva conduzida na China citada anteriormente apresentou especificidade de 100\%, assim como o estudo desenvolvido também na China realizado por Huang et al. (2021). Já na pesquisa prospectiva desenvolvida por Donovan et al. (2020) foi relatado que a especificidade do Xpert MTB/RIF Ultra utilizando um padrão de referência composto por mais de um teste foi de 100\%, mas, quando apenas a cultura foi usada como padrão de referência, a especificidade diminuiu ligeiramente para 93,9\%, valor semelhante ao encontrado no presente estudo (Shao et al., 2020; Huang et al., 2021; Donovan et al., 2020).

A diminuição da especificidade do Xpert MTB/RIF Ultra é esperada quando a cultura para micobactérias é utilizada como padrão de referência, visto que a cultura permite detectar apenas os bacilos viáveis e o Xpert MTB/RIF Ultra identifica também o DNA de bacilos não viáveis, não recuperados na cultura, gerando assim resultados do teste molecular aparentemente falso positivos contra um padrão de referência de cultura para micobactérias (Donovan et al. 2020; Arend \& Soolingen, 2017).

Uma segunda explicação para a diminuição da especificidade do Xpert MTB/RIF Ultra está associada à categoria traços. Essa categoria de rastreamento é resultado do aprimoramento do limite de detecção do Ultra e provavelmente colabora para a redução da especificidade do teste. O resultado positivo traços demonstra a detecção do DNA do M. tuberculosis em uma quantidade muito baixa. A Organização Mundial da Saúde (OMS) recomenda que a categoria traços, para amostras extrapulmonares, seja considerada resultado verdadeiro positivo para tomada de decisão clínica e acompanhamento dos pacientes, pois, segundo a OMS, o resultado positivo traço é suficiente para iniciar o tratamento, o que reforça a relevância do estudo em questão visto que o início do tratamento específico é fundamental e determinante para o indivíduo com meningite tuberculosa (Opota et al., 2019; Kohli et al., 2018; World Health Organization, 2017).

Nesse estudo, o Xpert MTB/RIF Ultra apresentou positividade em nove amostras que foram negativas na cultura e destas, seis foram categorizadas como traços. As possíveis justificativas para essa discordância entre os resultados da cultura e do Xpert MTB/RIF Ultra podem estar associadas a fatores já mencionados anteriormente, como o limite de deteç̧ão extrassensível do Ultra e a capacidade de detecção de bacilos viáveis e não viáveis.

Ao realizar uma análise detalhada no banco de dados do estudo dos pacientes que apresentaram resultado positivo no Xpert MTB/RIF Ultra e negativo na cultura foi possível observar que cinco apresentavam resultados anteriores positivos para M. Tuberculosis na cultura e/ou TRM-TB em outras amostras clínicas como escarro, lavado brônquico e aspirado traqueal. Sendo possível afirmar que os resultados positivos do Xpert MTB/RIF Ultra descritos no presente estudo seja consequência da presença do bacilo não viável na amostra analisada.

Uma preocupação relatada ao utilizar a versão anterior do Xpert MTB/RIF para o diagnóstico da meningite tuberculosa foi o VPN considerado inadequado para descartar meningite tuberculosa. Diante disso, vale destacar o alto VPN encontrado neste estudo para o Xpert MTB/RIF Ultra. Essa melhora relatada no Ultra está relacionada à sensibilidade aumentada e especificidade reduzida do teste que reflete em um VPN mais alto, porém um VPP mais baixo (Bahr et al., 2018; Arend et al., 2017).

Em uma pesquisa realizada no mesmo local do estudo em questão, no qual foi avaliado o desempenho do Xpert MTB/RIF para amostras pulmonares e extrapulmonares foi encontrada uma sensibilidade de $75 \%$ e especificidade de $100 \%$ para amostras de líquor quando considerada a cultura líquida como referência (Dietz et al., 2021). Fato que corrobora com os dados citados anteriormente em relação à melhora da sensibilidade do Xpert MTB/RIF Ultra, apesar da ligeira diminuição da especificidade.

Em estudos de avaliação de desempenho diagnóstico, a utilização de um padrão de referência adequado influencia diretamente nos resultados. Portanto, uma limitação encontrada nesse estudo diz respeito à utilização de um padrão de referência diferente, em relação às pesquisas encontradas na literatura, não sendo possível estabeceler uma comparação direta 
com as mesmas. Todavia, o diagnóstico da meningite tuberculosa é complexo e limitado, o que pode justificar a variação desses padrões de referências, não havendo uniformidade no padrão aplicado. Além disso, o padrão ouro para a tuberculose é a cultura líquida, entretanto neste estudo foi utilizada a cultura sólida em meio Lowenstein Jensen, constituindo dessa forma uma limitação.

\section{Conclusão}

O Xpert MTB/RIF Ultra apresentou uma sensibilidade e especificidade alta para o diagnóstico de meningite tuberculosa, apesar desta última ter sido reduzida ligeiramente. Esses resultados demonstram que o Xpert MTB/RIF Ultra apresenta melhorias que contribuem significativamente para a detecção dos casos de meningite tuberculosa.

Logo, a diminuição do tempo para obtenção do resultado e o aumento da sensibilidade, permitem que o tratamento apropriado para os pacientes com essa patologia seja iniciado mais rapidamente, melhorando o seu prognóstico. Diante disso, esse estudo corrobora com as recomendações da OMS quanto ao uso do Xpert MTB/RIF Ultra como teste de triagem para o diagnóstico de meningite tuberculosa.

Diante do número limitado de estudos, principalmente no que tange à utilização apenas da cultura como referência, a realização de estudos que definam o padrão de referência mais oportuno a ser empregado são necessários a fim de avaliar melhor o desempenho desse novo cartucho disponível no mercado.

\section{Referências}

Arend, S. M \& Soolingen, D. V. (2017). Performance of Xpert MTB/RIF Ultra: a matter of dead or alive. The Lancet Infectious Diseases, 18 (1), 8-10.

Bahr, N. C., Nuwagira, E., Evans, E. E., Cresswell, F. V., Bystrom, P. V., Byamukama, A., Bridge, S. C., Bangdiwala, A. S., Meya, D. B., Denkinger, C. M., Muzoora, C., Boulware, D. R., \& ASTRO-CM Trial Team (2018). Diagnostic accuracy of Xpert MTB/RIF Ultra for tuberculous meningitis in HIV-infected adults: a prospective cohort study. The Lancet Infectious diseases, 18(1), 68-75.

Bisognin, F., Lombardi, G., Lombardo, D., Re, M.C., \& Monte, P.D. (2018). Improvement of Mycobacterium tuberculosis detection by Xpert MTB/RIF Ultra: A head-to-head comparison on Xpert-negative samples. PloS One, 13(8), e0201934.

Chakravorty, S., Simmons, A. M., Rowneki, M., Parmar, H., Cao, Y., Ryan, J., Banada, P. P., Deshpande, S., Shenai, S., Gall, A., Glass, J., Krieswirth, B., Schumacher, S. G., Nabeta, P., Tukvadze, N., Rodrigues, C., Skrahina, A., Tagliani, E., Cirillo, D. M., Davidow, A., \& Alland, D. (2017). The New Xpert MTB/RIF Ultra: Improving Detection of Mycobacterium tuberculosis and Resistance to Rifampin in an Assay Suitable for Point-of-Care Testing. mBio, 8(4), e00812-17.

Chin, J. H., Musubire, A. K., Morgan, N., Pellinen, J., Grossman, S., Bhatt, J. M., Wadda, V., \& Ssengooba, W. (2019a). Xpert MTB/RIF Ultra for Detection of Mycobacterium tuberculosis in Cerebrospinal Fluid. Journal of clinical microbiology, 57(6), e00249-19.

Chin, J. H., Ssengooba, W., Grossman, S., Pellinen, J., \& Wadda, V. (2019b). Xpert MTB / RIF Ultra: Procedimentos ideais para a detecção de Mycobacterium tuberculosis no líquido cefalorraquidiano. Journal of Clinical Tuberculosis and Other Mycobacterial Disease, $14,16-18$.

Costa, R.R., Silva, M.R., \& Gonçalves, I.C. (2018). Diagnóstico laboratorial da tuberculose: Revisão de literatura. Revista Médica de Minas Gerais, 28 (5), eS280525.

Cresswell, F. V., Tugume, L., Bahr, N. C., Kwizera, R., Bangdiwala, A. S., Musubire, A. K., Rutakingirwa, M., Kagimu, E., Nuwagira, E., Mpoza, E., Rhein, J., Williams, D. A., Muzoora, C., Grint, D., Elliott, A. M., Meya, D. B., Boulware, D. R., \& ASTRO-CM team (2020). Xpert MTB/RIF Ultra for the diagnosis of HIV-associated tuberculous meningitis: a prospective validation study. The Lancet Infectious diseases, 20 (3), 308-317.

Dietz, J. C., Borges, M. S., Alves, S. L. A., Dias, D. X. R., \& Manrique, E. J. C. (2021). Desempenho do teste Xpert® MTB/RIF na detecção laboratorial de tuberculose em amostras pulmonares e extrapulmonares. Research, Society and Development, 10 (7), e36610716533.

Donovan, J., Thu, D., Phu, N. H., Dung, V., Quang, T. P., Nghia, H., Oanh, P., Nhu, T. B., Chau, N., Ha, V., Hang, V., Trinh, D., Geskus, R. B., Tan, L. V., Thuong, N., \& Thwaites, G. E. (2020). Xpert MTB/RIF Ultra versus Xpert MTB/RIF for the diagnosis of tuberculous meningitis: a prospective, randomised, diagnostic accuracy study. The Lancet Infectious diseases, 20(3), 299-307.

Garcia, M. L., Dantas, J. M. C., Malheiro, D. R., Moreira, Í. C., \& Lucena, M. M. (2016). Meningite Tuberculosa: Perfil epidemiológico no Brasil, no Ceará e no Cariri entre 2007 e 2015. Revista e-Ciência, 4(1).

Garg R. K. (2019). Microbiological diagnosis of tuberculous meningitis: Phenotype to genotype. The Indian journal of medical research, $150(5), 448-457$.

Huang, M., Wang, G., Sun, Q., Jiang, G., Li, W., Ding, Z., Jia, H., Gao, M., Huang, H., \& Li, Q. (2021). Diagnostic accuracy of Xpert MTB/RIF Ultra for tuberculous meningitis in a clinical practice setting of China. Diagnostic Microbiology and Infectious Disease, $100(1): 115306$. 
Kohli, M., Schiller, I., Dendukuri, N., Dheda, K., Denkinger, C. M., Schumacher, S. G., \& Steingart, K. R. (2018). Xpert ${ }^{\circledR}$ MTB/RIF assay for extrapulmonary tuberculosis and rifampicin resistance. The Cochrane database of systematic reviews, 8 (8), CD012768.

Opota, O., Mazza-Stalder, J., Greub, G., \& Jaton, K. (2019). The rapid molecular test Xpert MTB/RIF ultra: towards improved tuberculosis diagnosis and rifampicin resistance detection. Clinical Microbiology and Infection, 25(11), 1370-1376.

Pereira, A. S., Shitsuka, D. M., Parreira, F. J., \& Shitsuka, R. (2018). Metodologia da Pesquisa Científica. UFSM. https://repositorio.ufsm.br/bitstream/handle/1/15824/Lic_Computacao_Metodologia-Pesquisa-Cientifica.pdf?sequence=1.

Seddon, J. A., Tugume, L., Solomons, R., Prasad, K., Bahr, N. C., \& Tuberculous Meningitis International Research Consortium (2019). The current global situation for tuberculous meningitis: epidemiology, diagnostics, treatment and outcomes. Wellcome open research, 4 (167).

Seddon, J. A., Thwaites, G. E., \& Tuberculous Meningitis International Research Consortium (2019). Tuberculous meningitis: new tools and new approaches required. Wellcome open research, 4 (181).

Shao, L., Qiu, C., Zheng, L., Yang, Y., Yang, X., Liang, Q., Zhang, Y., Che, N., Pang, Y., \& Duan, H. (2020). Comparison of diagnostic accuracy of the GeneXpert Ultra and cell-free nucleic acid assay for tuberculous meningitis: A multicentre prospective study. International Journal of Infectious Diseases, 98, 441- 446 .

World Health Organization. (2017). WHO meeting report of a technical expert consultation: non-inferiority analysis of Xpert MTB/RIF ultra compared to Xpert MTB/RIF. World Health Organization. https://apps.who.int/iris/bitstream/handle/10665/254792/WHO-HTM-TB-2017.04eng.pdf?sequence $=1$ \&isAllowed $=\mathrm{y}$

World Health Organization. (2021). Global tuberculosis report 2021. https://www.who.int/publications/i/item/9789240037021

Zhang, Y., Huang, J., \& Du, L. (2017). The top-cited systematic reviews/meta-analyses in tuberculosis research: A PRISMA-compliant systematic literature review and bibliometric analysis. Medicine, 96 (6), e4822. 